\title{
Align Technology shares new iTero findings and benefits
}

\author{
Align Technology shares findings from new clinical study that validates the significant benefits of the iTero Element \\ 5D Imaging System as an aid in detection and monitoring of interproximal caries lesions
}

- The study published in the Journal of Dentistry confirms that the iTero Element $5 \mathrm{D}$ imaging system* ${ }^{\star}$ with iTero NIRI (Near Infra-Red Imaging) technology is more sensitive than bitewing radiography in detecting early enamel lesions and comparable in detecting dentinal lesions

- iTero NIRI technology of the iTero Element 5D imaging system was $66 \%$ more sensitive than bitewing $\mathrm{X}$-ray for detection of interproximal lesions. ${ }^{* *}$

Align Technology, a leading global medical device company that designs, manufactures and sells the Invisalign system of clear aligners, iTero intraoral scanners and exocad CAD/CAM software for digital orthodontics and restorative dentistry, has announced the findings of a multi-centre clinical study, Reflected near-infrared light versus bite-wing radiography for the detection of proximal caries: a multicentre prospective clinical study conducted in private practices, published in the peer-reviewed Journal of Dentistry. ${ }^{1}$ The study validates and further demonstrates the significant benefits of the iTero Element 5D imaging system as an aid in detection and monitoring of interproximal caries lesions above the gingiva without harmful radiation.

The clinical study was designed to compare the detection of interproximal caries by dentists using near infra-red technology (NIRI) and bitewing radiography. The results demonstrated high accuracy ( $\mathrm{p}<0.0001)$ detection of early enamel lesions $(88.6 \%)^{\star * *}$ and of carious lesions involving the dentino-enamel junction $(96.9 \%))^{* * *}$

In addition, the study compared NIRI and bitewing radiography to visual caries debridement. When compared against clinical evaluation of posterior proximal lesions observed during caries debridement, the NIRI technology of the iTero Element 5D imaging system was $66 \%^{\star *}$ more sensitive than bitewing $\mathrm{x}$-ray technology and demonstrated $96 \%^{* *}$ sensitivity for posterior interproximal lesions detections.

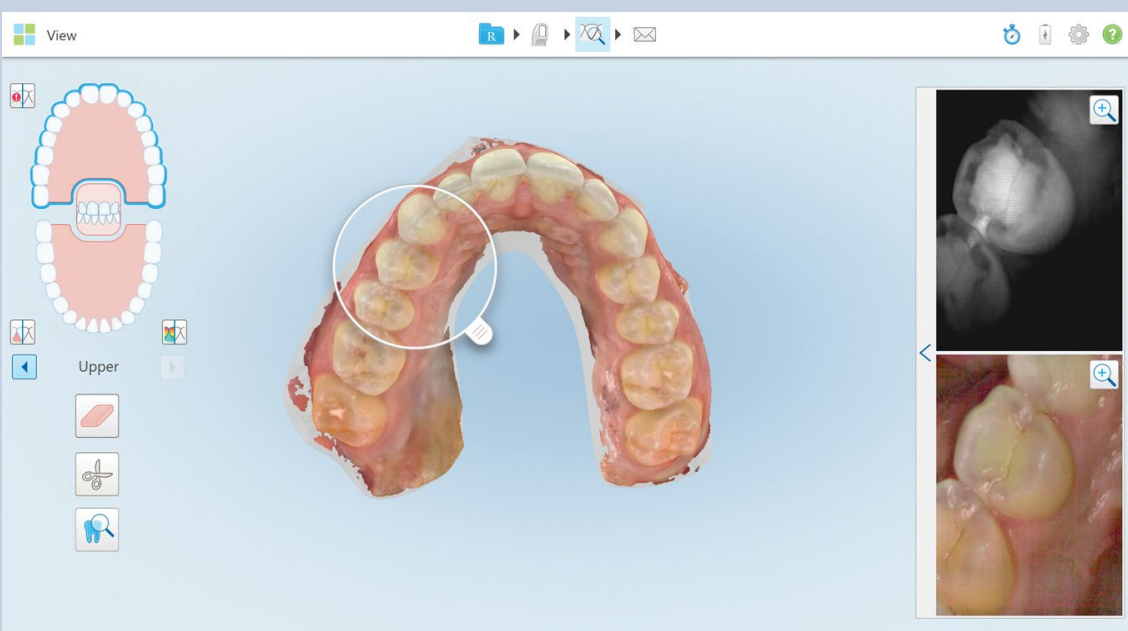

Yuval Shaked, senior vice president and managing director, iTero scanner and services business, Align Technology, said: 'We are pleased to see the results of these clinical findings further validating what doctors and their patients have experienced. The visualisation capabilities of the iTero Element 5D imaging system helps in the early detection of cavities without $\mathrm{x}$-ray radiation. This study emphasises the valuable role that the iTero 5D imaging system with NIRI technology is already providing doctors and their staff to support their dental assessments of patients and overall patient oral healthcare and treatment options. When combined with the ease of use and comfortable experience for a broad population of patients, the iTero $5 \mathrm{D}$ imaging system with NIRI technology is an essential tool for any doctor's office'.

As part of the study, the iTero Element $5 \mathrm{D}$ imaging system was used to perform intraoral scans on 100 patients in five dental clinics across Germany and Canada. The iTero Element 5D imaging system includes NIRI technology that scans the internal structure of a tooth (enamel and dentine) in real time that aids in caries detection while simultaneously capturing 3D colour images of dentition. Reflected near infra-red light, images of posterior teeth were used to detect interproximal caries and the results were then compared to bitewing radiography. NIRI was found to be more sensitive than bitewing radiography in detecting early enamel lesions and comparable in detecting dentinal lesions.

Lead study author Dr Zvi Metzger, Professor, Departments of Oral Biology and Endodontology, at The Goldschleger School of Dental Medicine, Tel Aviv University, said: 'Reflected near infra-red light images generated simultaneously during 3D scanning of dental arches with the iTero Element 5D imaging system scanner may be used reliably for detection, screening and monitoring of proximal caries. This method for caries detection may potentially minimise the traditional use of ionising radiation'.

For more information visit https://iterohub.com.

The study described was sponsored by Align Technology.

* iTero NIRI technology is the same across the iTero Element 5D system and some configurations of iTero Element Plus imaging systems since the wand, optics and software are the same.

** Data on file at Align Technology, September 2021

*** Data on file at Align Technology, February 2021

\section{Reference}

1. Metzger Z, Colson D G, Bown P, Weihard T, Baresel I, Nolting $T$. Reflected near-infrared light versus bite-wing radiography for the detection of proximal caries: A multicenter prospective clinical study conducted in private practices. J Dent 2021; doi: 10.1016/j. jdent.2021.103861. 